C2006 IEEE. Personal use of this material is permitted. However, permission to reprint/republish this material for advertising or promotional purposes or for creating new collective works for resale or redistribution to servers or lists, or to reuse any copyrighted component of this work in other works must be obtained from the IEEE. 


\title{
Defining Reputation in Service Oriented Environment
}

\author{
Farookh Khadeer Hussain ${ }^{1}$, Elizabeth Chang ${ }^{1}$ and Tharam S. Dillon ${ }^{2}$ \\ ${ }^{1}$ School of Information Systems \\ Curtin University of Technology \\ PO Box U1987 \\ Perth, WA, Australia 6845 \\ \{Farookh.Hussain, Elizabeth.Chang\} @ cbs.curtin.edu.au \\ ${ }^{2}$ Faculty of Information Technology \\ University of Technology, Sydney \\ Sydney, NSW 2007, Australia \\ tharam@it.uts.edu.au
}

\begin{abstract}
Reputation has a profound impact on the Trusting Agent and Trusted Agent in business interactions. Moral, ethical and legal guidelines are implemented as a result of the promotion of fair trading practices, honesty from all parties, consumer protection legislation, service quality assessment, and assurance for customers, e-businesses and service-oriented environments.

In this paper we propose a definition of reputation that is more suited to service oriented environments. Additionally we explain in detail, all the terms in the definition.
\end{abstract}

\section{Introduction}

Reputation has a profound impact on the Trusting Agent and Trusted Agent in business interactions. Moral, ethical and legal guidelines are implemented as a result of the promotion of fair trading practices, honesty from all parties, consumer protection legislation, service quality assessment, and assurance for customers, e-businesses and service-oriented environments.

In order to understand this leading edge technology, we first need to understand the concept of Reputation along with Reputation Query and the three inner relationships of reputation, which are: the Recommendation Trust Relationship, the Reputation Query Relationship and the Third Party Trust
Relationship. We also need to distinguish between Third Party Agents, Recommendation Agents, Known Agents, Referred Agents, Unknown Agents, Malicious Agents and Un-Trusted Agents. We shall examine $1^{\text {st }}$, $2^{\text {nd }}$ and $3^{\text {rd }}$ hand opinions, the Trustworthiness of Recommendation Agents to provide opinions (also known as credibility of Recommendation Agent) and the Trustworthiness of the Opinion. We learn how to modify the Trustworthiness value of the Recommendation Agent to provide an opinion, and finally we present the Reputation Model for a better and more adequate Agent, product and service assessment. The above ethical fundamentals are the foundations of a customer's perceptions of Business and Service integrity.

Some technologies treat human networks (both users who are registered on their website or customers of the site) as trustworthy, and others (outsiders, or unknown Agents) as untrustworthy [1]. The questions of trust in networked environments include issues concerning interactive relationships within these environments such as someone who is known can be untrustworthy, and someone who is known may be trustworthy in one context (e.g. work on projects) and untrustworthy in another (e.g. promotion as they become competitors in this context).

Another question that could arise is, "If it is someone we do not know, are they trustworthy or untrustworthy?". It can not simply be stated that unknown people are all untrustworthy or malicious, especially with billions of existing Internet users and a large client or user population that is unknown. This is 
unlike the physical world, where our immediate social sphere is much smaller (e.g. in an organization of 100 people, all of which are known Agents to each other, it might be assumed that $2 \%$ of them are malicious, or untrustworthy). However, in the virtual world our reach to known and unknown Agents is beyond comprehension in conventional trust relationships.

Another problem remains in relation to trust and reputation where agents can sometimes be inconsistent over time. How do we manage the need to distinguish malicious Agents from untrustworthy Agents? Our understanding of Trustworthy Agents is that they may actually be untrustworthy in some time slots and contexts. Our final considerations may be the trust approach towards unknown Agents and the level of trust we place in Third Party opinions regardless of whether they are known to us or not.

In this paper we take these concerns into consideration. We explain the complex concepts and terminologies one by one. We define reputation in a form suitable for service-oriented environments and ebusiness. Existing definitions will also be reviewed. A new set of terms that comes with the notion of reputation will be examined. We also focus on the differences between Trust and Reputation including distinguishing between trust relationships and reputation relationships.

The key complication in Reputation and Reputation Relationships is the involvement of Third Party Agents. We take the reader step by step through the complexities of the reputation relationship including the reputation query relationship, recommendation trust relationships and Third Party Trust Relationships. We also separate the Third Party Trust Agents into Known Agents, Referred Agents, Unknown Agents and Malicious Agents. Furthermore, we explain the need to consider $1^{\text {st }}$ hand, $2^{\text {nd }}$ hand and $3^{\text {rd }}$ hand opinions and how a reputation value should be aggregated. We also develop Reputation Models using these advanced reputation concepts, all of which are for the purpose of a more adequate and fair assessment of Agents, products, services and quality assurance for end users and consumers. In the next section, we provide a definition of reputation.

\section{Definition of Reputation}

Definition 1a: In service-oriented environments, we define agent reputation as an aggregation of the recommendation from all of the Third Party Recommendation Agents, in response to the Trusting Agent's Reputation Query about the Quality of the
Trusted Agent in a given context and at a given point in time.

The definition also applies to the Reputation of the quality of product and service.

Definition 1b: In service-oriented environments, we define product reputation as an aggregation of the recommendation from all of the Third Party Recommendation Agents, in response to the Trusting Agent's Reputation Query about the Quality of the Product (QoP), in a given context and at a given point in time.

Definition 1c: In service-oriented environments, we define service reputation as an aggregation of the recommendation from all of the Third Party Recommendation Agents, in response to the Trusting Agent's Reputation Query about the Quality of the Service (QoS), in a given context and at a given point in time.

There are four key primary concepts in the reputation definition:

1) Reputation: aggregation of all the recommendations from the Third Party Recommendation Agents about the quality of the Trusted Agent.

2) Recommendation (including opinion and recommendation value): submitted by the Third Party Recommendation Agents (Recommenders or Witness Agents).

3) Recommendation Agent: submit the recommendation or opinion to the Trusting Agent or respond to a reputation query.

4) Reputation Query: a query made by the Trusting Agent about the Trusted Agent in a given context and time slot.

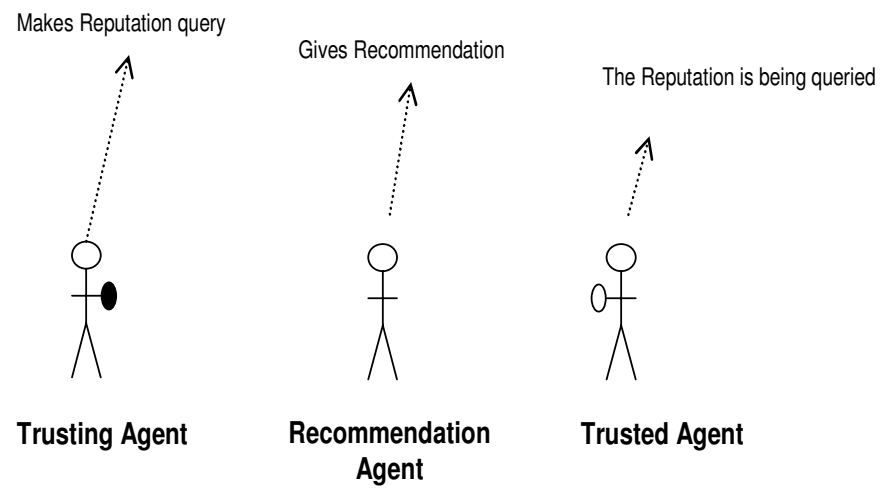

Figure 1 Three Key entities in Reputation Definition

The terms 'Reputation Query', 'Third Party Recommendation Agents', 'Trustworthiness of Recommendation Agent', 'Trusting Agent', 'Trusted Agent are essential when defining Reputation. These 
new terms can be regarded as the building blocks of Reputation, particularly in service-oriented environments. These new terms introduced in the definition of reputation make a fundamental distinction between Trust and Reputation.

In this paper, we will pay more attention to the new terms, new concepts and will distinguish between Trust and Reputation.

Note that in this paper we use the following terms interchangeably, namely: 'the Third Party Recommender' is a synonym to the 'Recommendation Agent' and 'Recommender' and 'Witness Agent'. 'Making a recommendation' is synonymous with 'giving an opinion', and 'recommendation' is synonymous to 'opinion'. Trustworthiness of Recommendation Agent to give an opinion and credibility of Recommendation agent are synonymous and used interchangeably.

\subsection{Third Party Recommendation Agents}

In service-oriented network environments the $3^{\text {rd }}$ Party Recommendation Agents are those agents who provide a recommendation, feedback or opinion in response to the reputation query issued by the trusting agent. The recommendation could be about a trusted agent, product or service. Sometimes we use the term Recommendation Agents or Recommender or Witness Agents to refer to Third Party Recommendation Agents.

\subsection{Reputation Query}

A Reputation Query is the enquiry made by the Trusting Agent about the trusted agent or product or service. A reputation query contains request for particular information about trusted agent, product or service. The reputation query contains different fields as like the context in which the trusting agent wants to enquire about the trusted agent or service or product. Additionally it contains the time at which the trusting agent wants to know the trustworthiness of the trusting agent, product or service.

- In regard to a Trusted Agent, the enquiry is about the trustworthiness of the Trusted Agent in a given context and at a given timeslot.

- In regard to a Product, the enquiry is about the Quality of the Product in a given context of the product.

- In regard to a Service, it is a request for feedback about the Quality of Service in a given context of the service.
The term Reputation Query is also interchangeable with 'asking opinions', 'getting recommendations', 'calling for referees', 'soliciting recommendations' or 'an invitation for feedback'. The Reputation Query has the following characteristics:

- A reputation query is issued by exactly one Trusted Agent, also known as the Reputation-Querying Agent. An example of Reputation Query is request for information for product SDF-123 such as 'The Quality of Laptop SDF-123, manufactured in 2005, in terms of the life of its battery'. The above mentioned reputation query contains request for information about a product (laptop SDF-123), just one context about the product (life of the laptop's batteries) at a given time slot (in 2005).

The reputation querying agent can issue a reputation query that can contain request for information about a given trusted agent, product or service in more than one context and more than one time slot. Additionally a reputation query may contain request for information about more then one trusted agent or product or service. An example of such reputation queries are as follows

(a) 'The Quality of Laptop SDF-123, manufactured in 2005, in terms of the life of its battery and the screen resolution'. This reputation query contains request for information about SDF-123 in more than one context.

(b) 'The Quality of Laptop SDF-123, manufactured in 2003 and 2005, in terms of the life of its battery. This reputation query contains request for information about SDF-123 a single context at more then one time slot.

(c) 'The Quality of Laptop SDF-123 and the Quality of Laptop SGH-145 each of them manufactured in 2003 and 2005, in terms of the life of its battery. This reputation query contains request for information about SDF-123 and SGH-145 in a single context at more then one time slot.

- $\quad$ The reputation query is responded to by zeroto-many Third Party Agents. It may be associated with zero-to-many (0:M) Third Party Agents who respond to the reputation query. A reputation query may result in no response at all.

In case, the reputation query, requests for information for more than one product/ agent or service, then the $3^{\text {rd }}$ Party Agent should reply about the agent/ product or service about which it knows. If case the reputation query requests for information about a given agent / product / service in more than one context, then the $3^{\text {rd }}$ Party Agent should reply about the context of the agent/ service / product which it knows. If case the reputation query requests for information 
about a given agent / product / service in more than one timeslot, then the $3^{\text {rd }}$ Party Agent should reply about the timeslot of the agent/ service / product which it knows in which it has previously of the agent/ product or service about which it knows. A given $3^{\text {rd }}$ Party Agent may not know the trustworthiness / QoS / QoP of the agent / service / product in all the context and time slot specified in the reputation in order to reply about a given context or time slot.

- Similar reputation queries may be posted by many Trusting Agents. For example, several Agents want to know details about 'The quality of service provided by $\mathrm{ABC}$ Logistics in 'Transferring goods from Perth to Sydney'.

- $\quad$ Each reputation query can be issued for one or more trusted agents or products or services. The reputation query is further qualified by the context and a given timeslot of the trusted agents or products or services. The context in the reputation query is specific to an Agent, a product or a service.

- The context is a key component in Reputation Query. As mentioned before the context specifies the scenario for which the Trusting Agent wants to solicit trustworthiness/ QoS/ QoP of the Trusted Agent/ Product or Service. For each Trusted Agent or Product or Service specified in the reputation query there has to be at least one context about the reputation queried agent.

- The timeslot is a key component in Reputation Query. As mentioned before the timeslot specifies the time for which the Trusting Agent wants to solicit trustworthiness/ QoS/ QoP of the Trusted Agent/ Product or Service. For each Trusted Agent or Product or Service specified in the reputation query there has to be at least one timeslot about the reputation queried agent.

A reputation query may involve context that refers to negative activities or positive activities about the Trusted Agent.

\subsection{Trusting Agent in a Reputation}

A Trusting Agent in a reputation query is an agent who poses a query, requests recommendation or asks feedback or seeks references about the Trusted Agent or entity from $3^{\text {rd }}$ Party Recommendation Agents on the network. The $3^{\text {rd }}$ Party recommendation Agent is also known as Reputation Querying Agent.

If a Trusting Agent wants to carry out a transaction with a Trusted Agent, but she or he has not interacted with the Trusted Agent previously, the Trusting Agent is to ask for recommendations from other Agents (Third Parties) about their past experiences with the
Trusted Agent, in order to decide whether or not to carry out a transaction with the Trusted Agent or choose to use the Trusted Agent.

\subsection{Trusted Agent in a Reputation}

The term Trusted Agent used in defining reputation is a generic one and could refer to any entity about whom the trusting agent is interested in. It could be an intelligent software agent, a product, a service, a website, etc that the trusting agent is interested in.

The Trusted Agent in a reputation query is also known as the Reputation-Queried Agent whose reputation is under consideration or evaluation.

\subsection{Trustworthiness of Opinion or Witness Trustworthiness Value (WTV)}

The trustworthiness of an opinion or witness trustworthiness value is defined as a numeric value that denotes or quantifies the correctness of an opinion communicated by the $3^{\text {rd }}$ Party Recommendation Agent.

The trustworthiness of an opinion is held by the trusting agent about the $3^{\text {rd }}$ Party Recommendation Agent and it denotes the correctness of recommendations communicated by the 3rd Party Agent to the trusting agent.

From the perspective of the trusting agents, the $3^{\text {rd }}$ Party Agents are classified into the following two types based on the correctness of recommendations communicated by the $3^{\text {rd }}$ Party recommendation agent.

1) Known Agents: We define a known agent as an agent whose witness trustworthiness value is known to the trusting agent.

The trusting agent had previously solicited recommendations from the agent in question and based on previous interaction/s the trusting agent knows the witness trustworthiness value (or rather the correctness of recommendations communicated by the $3^{\text {rd }}$ party Agent).

2) Unknown Agents: We define a known agent as an agent whose witness trustworthiness value is not known to the trusting agent.

The trusting agent has not previously solicited recommendations from the agent in question hence knows the witness trustworthiness value (or rather the correctness of recommendations communicated by that agent). 
From the perspective of the trusting agents, the Known $3{ }^{\text {rd }}$ Party Agents are further classified into the following two types:

a. Trustworthy Known Agents: We define a known a trustworthy known agent as an agent whose witness trustworthiness is with in a specified range or value. The specified value range of witness trustworthiness values for $3^{\text {rd }}$ party Agent to be considered as trustworthy is usually specified by the trusting agent.

In business terms, a trustworthy known agent is an agent from whom the trusting agent had previously taken recommendations and the witness trustworthiness value of this agent based on these previous recommendations falls with in a given range of witness trustworthiness values. The trusting agent considers each agent falling with in this range as trustworthy known agent or trustworthy witness agent or trustworthy recommender.

b.Untrustworthy Known Agents: We define a known an untrustworthy known agent as an agent whose witness trustworthiness is outside the specified range of witness trustworthiness values.

In business terms, a trustworthy known agent is an agent from whom the trusting agent had previously taken recommendations and the witness trustworthiness value of this agent based on these previous recommendations does not fall with in a given range of witness trustworthiness values. The trusting agent considers each agent falling with in this range as untrustworthy known agent or untrustworthy witness agent or untrustworthy recommender.

The concept of 'Giving an Opinion or a Recommendation' is interchangeable with 'writing a review' or 'offering feedback' about the Trusted Agent or product or service. Therefore, we sometimes treat the above words as the same and use them interchangeably.

\subsection{Quality of Agent, Quality of Product or Quality of Service}

Trustworthiness of an agent / product / service quantifies and expresses the quality of an agent or service or product. Trustworthiness of an Agent, service or a product in the service-oriented environment implies the "quality" of an Agent, service or product. The quality of a given service in serviceoriented environments is determined by determining the correlation between (a) The delivered quality of service (and)

(b) The mutually agreed quality of service.

We define the delivered service as the set of all functionalities that the trusted agent has delivered to the trusting agent. The delivered quality of service is a numeric value that quantifies and expresses, in commonly used terminology the value of all the delivered functionalities.

We define the mutually agreed service as the set of all functionalities that the trusted agent has promised to deliver to the trusting agent. The mutually agreed quality of service is a numeric value that quantifies and expresses, in commonly used terminology the value of all the mutually agreed functionalities.

The quality of a given product in service-oriented environments is determined by determining the correlation between

(i) The delivered value of the product (and)

(ii) The mutually agreed value of the product

We define the delivered value of a product as a numeric value that quantifies and expresses, in commonly used terminology the value of all the delivered functionalities of the product.

We define the mutually agreed value of a product as a numeric value that quantifies and expresses, in commonly used terminology the value of all the mutually agreed functionalities of the product.

The trustworthiness of an agent in service-oriented environments is determined by determining the correlation between

(1) The actual behaviour of the trusted agent in the interaction (and)

(2) The mutually agreed behaviour of the trusted agent in the interaction

We define the actual behaviour of the trusted agent as the set of all the functionalities that the trusted agent has delivered to the trusted agent in the interaction.

We define the mutually agreed behaviour of the trusted agent as the set of all the functionalities that the trusted agent has agreed to deliver to the trusted agent in the interaction.

\section{Conclusion and Further Work}

In this paper we proposed a definition of reputation. Additionally, we explained in detail the concept of 'agent reputation', 'service reputation' and 'product reputation'. 
The terms of 'Third Party Recommendation Agents', 'Witness Trustworthiness Values', 'Trusting Agent', 'Trusted Agent' and 'Reputation Query', which have been undefined in the literature have been dealt in depth in this paper.

Further work involves determining the inner relationships with the reputation relationship. Additionally further work involves developing ontology for trust and reputation.

\section{References}

[1] Chang, E., Dillon, T.S., Hussain, F.K., 'Trust and Reputation in Service Oriented Environment', John Wiley and Sons. (To be published in 2005). 